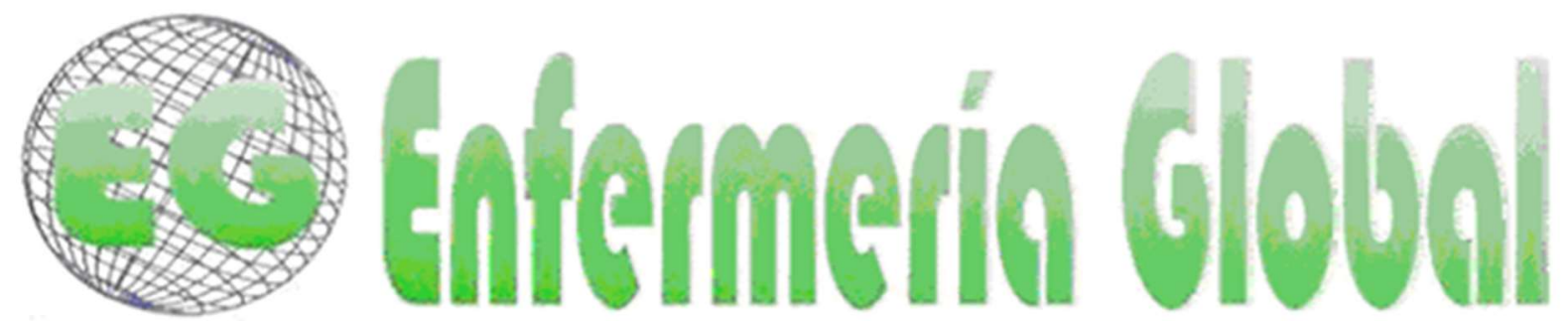

\title{
ORIGINALES
}

\section{Estrés en graduados y técnicos en cuidados auxiliares de enfermería en las áreas quirúrgicas hospitalarias}

Stress in nursing graduates and healthcare assistants in surgical areas of hospital

\author{
María de los Ángeles Ferraz Mesa ${ }^{1}$ \\ María del Carmen Serrano Barberá ${ }^{2}$ \\ María José López Montesinos ${ }^{3}$ \\ Laura Martínez Alarcón ${ }^{4}$ \\ Jesús María González Martín ${ }^{5}$
}
${ }^{1}$ Doctora en Enfermería. Enfermera asistencial, Hospital Universitario de Gran Canaria Doctor Negrín. Las Palmas de Gran Canaria. España. angeles fm@msn.com
2 Doctora en Enfermería por la Universidad de Murcia. Murcia. España.
${ }^{3}$ Doctora en Enfermería. Profesora Universidad de Murcia. Murcia. España.
${ }^{4}$ Profesora Asociada, Universidad de Murcia. Murcia. España.
5 Matemático, Unidad de Investigación del Hospital Universitario de Gran Canaria Doctor Negrín. La Palmas de Gran Canaria. España.

\section{https://doi.org/10.6018/eglobal.423851}

Recibido: 20/04/2020

Aceptado: $24 / 07 / 2020$

\section{RESUMEN:}

Objetivos: Analizar el nivel de estrés que presentan los Graduados de Enfermería y los Técnicos en Cuidados Auxiliares de Enfermería en las áreas quirúrgicas de los Hospitales Públicos de Gran Canaria.

Material y método: Estudio cuantitativo, descriptivo/observacional de prevalencia transversal, de 143 sujetos (Grado de Enfermería y Técnicos en Cuidados Auxiliares de Enfermería, en adelante T.C.A.E.), en Hospital Universitario de Gran Canaria Doctor Negrín, en adelante H.U.G.C. Dr. Negrín, y Complejo Hospitalario Universitario Insular-Materno Infantil de Canarias, en adelante C.H.U. Insular-Materno Infantil de Canarias, en los servicios de Quirófano, Unidad de Reanimación y Cuidados Críticos (R.E.A.), Unidad de Recuperación Post-anestésica (U.R.P.A.) y Cirugía Mayor Ambulatoria (C.M.A.), durante los años 2016-2019. Mediante una encuesta autocumplimentada de carácter anónimo y voluntario formado por la Escala de estrés percibido (PSS) de Cohen, S., Kamarck, T., y Mermelstein, R. Dispuesta en 14 ítems, además se realizó un cuestionario de 19 ítems de carácter demográfico y sociolaboral, efectuándose cruce de variables dependientes e independientes.

Resultados: Presentan un nivel de estrés con una mediana de $22 \%$. La media es 21.79 , la desviación típica 7.26 , el percentil 25 da como resultado $17 \%$, el percentil 50 es $22 \%$ y el percentil 75 es $39 \%$. La fiabilidad total da como resultado 0.86 en el Alfa de Cronbach. 
Conclusiones: Los trabajadores TCAE y Graduados de Enfermería, de las unidades mencionadas presentan un nivel moderado de estrés, pero las instituciones deberían intervenir para minimizarlo lo máximo posible.

Palabras clave: estrés laboral, enfermería, afrontamiento del estrés, ansiedad, estrés.

\begin{abstract}
:
Objectives: To analyse the stress level in Nursing Graduates and Healthcare Assistants in surgical areas of the Public Hospitals in Gran Canaria.

Material and Methods: Quantitative, descriptive/observational study of cross-sectional prevalence in 143 subjects (Nursing Degree and Healthcare Assistant, henceforth (H.C.A.), in the University Hospital of Gran Canaria Doctor Negrín, henceforth U.H.G.C. Dr. Negrín and Complejo Hospitalario Universitario Insular-Materno Infantil de Canarias(Children and maternity care hospital of the Canary Islands), henceforth the C.H.U. Insular-Materno Infantil de Canarias, in the surgery services/operating rooms, Trauma Resuscitation Unit (TRU), Post-Anesthesia Care Unit (P.A.C.U.), and Major Outpatient Surgery (M.O.S.), from 2016 to 2019. By means of an anonymous and voluntary self-completion survey based on the Perceived Stress Scale (PSS) from Cohen, S., Kamarck, T., and Mermelstein, R. It was distributed in 14 items, a questionnaire of 19 demographic and socio-labour items was also carried out, applying a cross-sectional design of dependent and independent variables.

Results: They show a stress level with a median of $22 \%$. The mean is 21.79 , the standard deviation is 7.26 , the 25 th percentile results in $17 \%$, the 50 th percentile is $22 \%$ and the 75 th percentile is $39 \%$. Total reliability is 0.86 using Cronbach's Alpha.

Conclusions: H.C.A. workers and Nursing Graduates from the aforementioned units show a moderate level of stress, but institutions should intervene to minimize it as much as possible.
\end{abstract}

Key words: work stress, nursing, dealing with stress, anxiety, stress.

\title{
INTRODUCCIÓN
}

Como antaño, entre los objetivos que desea alcanzar la enfermería actual, se encuentra proporcionar una atención de calidad al paciente/cliente, razón de ser de nuestra profesión, para ello es imprescindible que el profesional esté en un clima laboral propicio y minimizar el nivel de estrés, pero está siendo muy afectado por la alta prevalencia de estrés laboral, y el personal de enfermería actualmente experimenta niveles más altos de agotamiento relacionado con el estrés en comparación con otros profesionales de Ciencias de Salud.

La Organización Mundial de la Salud (O.M.S.) ${ }^{(1)}$, define el estrés como "el conjunto de reacciones fisiológicas que prepara al organismo para la acción". El inicio del estrés viene de la mano de los inicios de la humanidad, nos remontamos a sus comienzos, cuando enfrentarse a una situación de estrés tenía como respuesta una reacción de lucha o huida. En definición actualizada "es la respuesta automática y natural de nuestro cuerpo ante las situaciones que nos resultan amenazadoras o desafiantes". El estrés se produce como consecuencia del desequilibrio entre las demandas de la situación (estresores internos o externos) y los recursos de las personas (2). Este proceso es perfectamente reconocible en el ámbito laboral, especialmente cuando se da en trabajadores cuya profesión se desarrolla de forma intensa y constante en contacto con otras personas.

En cuanto a los estresores se pueden definir como cualquier suceso, situación, persona $u$ objeto que se percibe como estímulo o situación que provoca una respuesta de estrés en la persona evaluada. "Cualquier evento que cause impacto o impresión en el organismo humano." 
"El estrés psicológico es el resultado de una relación particular entre el individuo y el entorno que es evaluado por este como amenazante o desbordante de sus recursos y pone en peligro su bienestar", por tanto, la manera en que la persona aprecia una situación determinará que sea percibida como estresante ${ }^{(2)}$. Además, introducen como nuevo término "el afrontamiento del estrés" y una evaluación de estrés en tres tipos. Evaluación primaria, en ella se evalúan las demandas, categorizando un acontecimiento como irrelevante, benigno-positivo o estresante. El estresante a su vez puede ser de cuatro tipos (Amenaza: cuando se anticipa un daño o pérdida. Daño-pérdida: cuando ya se ha recibido un perjuicio. Desafío: implica una ganancia positiva y una amenaza. Beneficio: es una valoración positiva que no lleva al estrés). En la evaluación secundaria se valoran los propios recursos para afrontar la situación. Organización de la acción: se establece el comportamiento motor a llevar a cabo para afrontar las demandas, así como movilizar los recursos cognitivos y fisiológicos necesarios para poder llevar a cabo esas conductas.

Se desarrolló un modelo denominado enfoque psicosocial de estrés o enfoque de los acontecimientos vitales. Se crearon listados de los sucesos vitales más estresantes, examinando más de 5.000 registros médicos, elaborándose un listado de 43 acontecimientos vitales al que llamamos "Escala de Estrés de Holmes y Rahe" (3).

Los sucesos vitales son experiencias objetivas que alteran o amenazan las actividades cotidianas del individuo, causando un reajuste sustancial de su conducta.

Se ha comprobado que las molestias diarias y las dificultades crónicas pueden ser tan estresantes como los acontecimientos vitales mayores, además pueden ejercer un gran impacto sobre el bienestar físico y psicológico" (4), aunque dependerá de cómo el individuo tiende a pensar en términos negativos ${ }^{(5)}$.

Con respecto a las fuentes de estrés, provienen del entorno, el propio cuerpo y los propios pensamientos.

- Entorno: el medio, los ruidos, aglomeraciones, horarios, etc.

- Cuerpo: enfermedades, accidentes, etc.

- Pensamientos: manera de interpretar y catalogar las experiencias, el modo de ver el futuro, etc.

En cuanto a la respuesta de estrés en el organismo, puede darse a distintos niveles, cada persona responde de una forma diferente y no de manera estática según su evolución y las estrategias distintas que va desarrollando en su vida para enfrentar el estrés. A pesar de tantos años de estudio, el estrés continúa investigándose en el área de la salud, siendo considerado como "la enfermedad del siglo veinte" (6).

Por todo esto el objetivo de este estudio se centraba en conocer el nivel de estrés del personal de Grado de Enfermería y Técnicos en Cuidados Auxiliares de Enfermería, en adelante T.C.A.E. e identificar si el estrés está asociado a las variables sociolaborales y demográficas de la muestra. 


\section{MATERIAL Y MÉTODO}

Ha sido un estudio cuantitativo, descriptivo/observacional de prevalencia (trasversa). El cuestionario se administró por el entrevistador, con escalas Likert dicotómicas y policotómicas y la muestra se obtuvo por muestreo causal o incidental.

En cuanto al universo de la muestra a estudio ha sido de 457 sujetos, Este número es la sumatoria del personal, que ha sido aportado por los supervisores de enfermería de ambos hospitales, mediante la sumatoria de cada una de las áreas, en los servicios de Quirófano, Unidad de Reanimación y Cuidados Críticos, en adelante R.E.A., Unidad de Recuperación Post-anestésica, en adelante U.R.P.A. y Cirugía Mayor Ambulatoria, en adelante C.M.A. Se han obtenido 151 encuestas autocumplimentadas, a las que se le ha aplicado los criterios de inclusión y exclusión quedando un total de 143 sujetos, siendo este el tamaño de la muestra a estudio.

Los criterios de inclusión son:

- Trabajar en las fechas de la recogida de datos en los servicios de Quirófanos, REA, CMA y URPA en las categorías de Enfermería y TCAE.

- Tener una experiencia mayor de un mes en los servicios señalados.

- Estar dedicados exclusivamente a la asistencia del paciente (se excluyen personal que realicen otras tareas cómo formación, supervisión, etc.).

Los criterios de exclusión son:

- Negativa a participar en el estudio.

- Cuestionarios en blanco o mal cumplimentados.

Según la relación entre el número de ítems por constructo y tamaño de la muestra de esta es representativa (7). Los conglomerados estuvieron representados por los servicios donde los profesionales trabajaban. El estudio se llevó a cabo durante los años 2016-2019.

Tras la revisión bibliográfica efectuada, para conocimiento y análisis de la situación, se llevó a cabo un trabajo de campo para medir el nivel de estrés en el puesto de trabajo, en cuanto a la actividad de los profesionales de enfermería (Grado de Enfermería y T.C.A.E.), en los dos Hospitales públicos de la isla de Gran Canaria, El Hospital Universitario de Gran Canaria Doctor Negrín, en adelante H.U.G.C. Dr. Negrín y Complejo Hospitalario Universitario Insular-Materno Infantil de Canarias, en adelante C.H.U. Insular-Materno Infantil de Canarias, en las unidades de Quirófano, R.E.A., U.R.P.A. y C.M.A. y se realizó análisis y tratamiento de los datos.

Con respecto a la metodología utilizaremos como herramientas de medición un cuestionario con variables demográficas y sociolaborales, como variables independientes, dispuesto en 19 ítems, así como un cuestionario con variables dependientes, validado documentalmente, la Escala de estrés percibido (PSS) (8). Dispuesta en 14 ítems, tras obtener como referente diferentes artículos científicos ${ }^{(9)}$. Las dimensiones que mide este cuestionario son la percepción global del estado de estrés y la repuesta al estrés. 
El Análisis y tratamiento estadístico de datos se realizó con el programa Excel. Programa estadístico R Core Team 2018, versión 3.5.2. En cuanto al Análisis realizado fue Media, Desviación Típica, Mediana y Percentiles 25 y 75 en las variables cuantitativas. Frecuencia y Porcentaje en las variables cualitativas. Test de Alfa de Cronbach y Coeficiente de correlación de Spearman.

El estudio se realizó con la previa aprobación del Comité de Ética y Legislación (número del código del dictamen: 994/2018-027-1). Cumpliendo las normas de BCP (CPMP/ICH/135/95).

Referente al Estrés Laboral, se presenta en la mediana el valor $0 \%$, es el mejor valor, ya que se interpretaría como menor estrés y el valor máximo $56 \%$, como el máximo nivel de estrés, la mediana se sitúa en $22 \%$ por lo que se interpreta que tienen un nivel moderado de estrés, pero se tendría que intervenir en él para aproximarlo al menor nivel posible.

\section{RESULTADOS}

Los resultados de nuestro estudio presentan un nivel de estrés con una mediana de $22 \%$. La media es 21.79 , la desviación típica 7.26 , el percentil 25 da como resultado $17 \%$, el percentil 50 es $22 \%$ y el percentil 75 es $27 \%$. La fiabilidad total da como resultado 0.86 en el Alfa de Cronbach, el test de Normalidad de Shapiro-Wilk tiene una significación estadística $(p<0.337)$.

Con respecto a la edad de los encuestados (Gráfico 1) oscilan entre 29 y 64 años, siendo la media 43,57 años y la desviación típica 8,67. El percentil 25 está en 36 años, el percentil 50 en 43 años y el percentil 75 en 50 años. Se agrupan las edades en tres categorías comprendidas en: 29-38, 39-47, 48-64 años. De la población estudiada $81.82 \%$ son mujeres y $18,18 \%$ son hombres, respectivamente 26 hombres y 117 mujeres del total de la muestra $(\mathrm{N}=143)$. Las categorías laborales que se han estudiado han sido Grado de enfermería (se incluyen anteriores denominaciones de la titulación), el $63,64 \%$ y T.C.A.E. (se incluyen anteriores denominaciones de la titulación), el 33,36\%. Corresponde a 91 sujetos y 52 sujetos respectivamente.

En cuanto a la localización de los encuestados, o lugar de su puesto de trabajo (Gráfico 2), el 63.64\% fue personal del H.U. Dr. Negrín, mientras que en el C.H.U. Insular-Materno Infantil de Canarias fue un $36.6 \%$. La mayoría de los participantes trabajaba en la unidad de Quirófano $71.33 \%$ y en la unidad de menor participación fue C.M.A. con un $4.2 \%$, siendo R.E.A. y U.R.P.A. donde se encuentran los valores intermedios $16.08 \%$ y $8.39 \%$ respectivamente. No hay evidencias para afirmar que las medianas en hombres y mujeres son distintas, ni entre los dos Hospitales Públicos, ni entre las dos titulaciones estudiadas, se ha utilizado el test no paramétrico de la UMann-Whitney, como el p-valor es mayor de 0.05. Se ha utilizado el test de Jonckheere-Terpstra para comprobar si a medida que aumenta la edad (variable ordinal), aumenta o disminuye el nivel de estrés. Como el $p$-valor $=0.90$, es superior a 0.05 , no hay una tendencia que a mayor o menor edad hay más estrés. 
Gráfico 1.- Edad

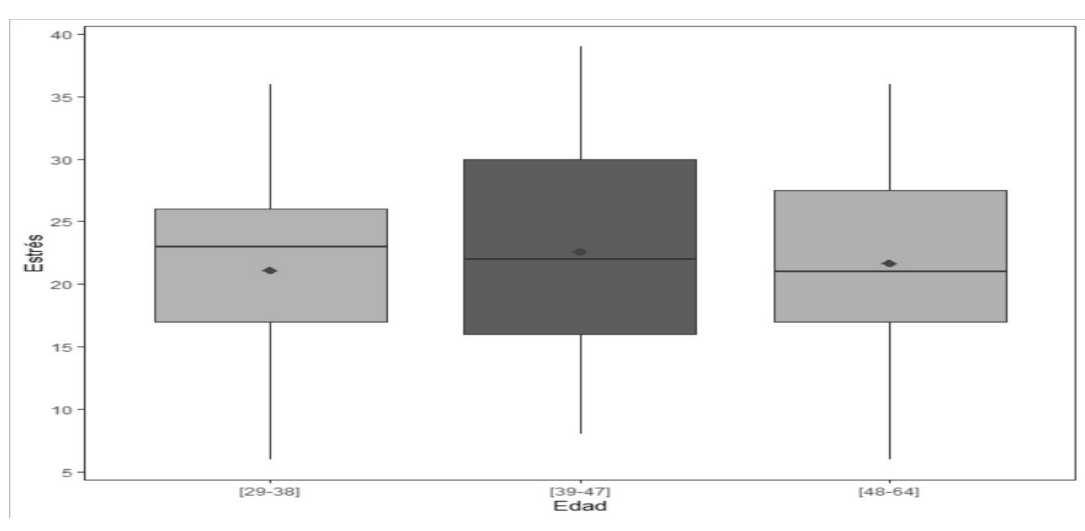

Gráfico 2.- Servicios de trabajo

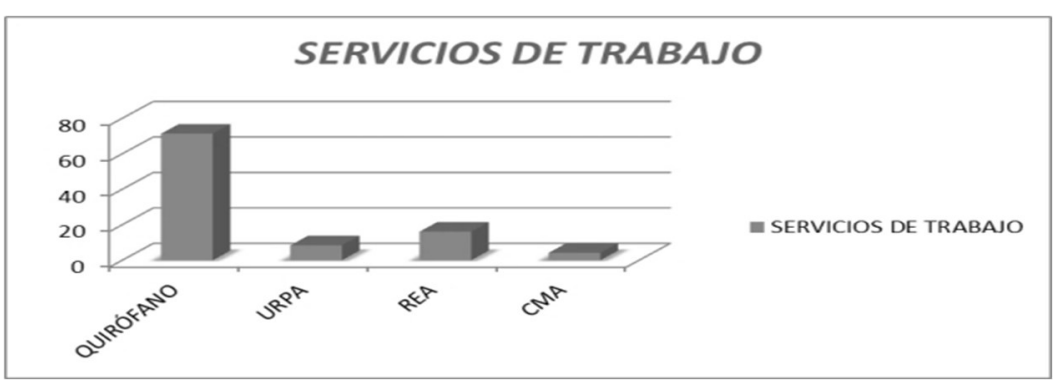

El $60.84 \%$ de los sujetos están casados o con pareja, de los que el $67.83 \%$ tienen hijos. Así mismo, el 33,57\%, la mayoría, no tiene hijos, el 31.47\% tiene dos, el 27,97\% tiene uno, el 6,29\% tiene tres y el $0.7 \%$ tiene cuatro. Según el Instituto Nacional de Estadística "el indicador Coyuntural de Fecundidad por provincia, Las Palmas, según orden de nacimientos, hijos por mujer en 2017 es de 1.05 hijos y en 2018 es de 0.99 hijos". En cuanto al número de hijos, la media en el grupo de mujeres Graduadas en Enfermería es 0.959 hijos y en el de T.C.A.E. es de 1.5 hijos. Se puede observar que este número en Graduadas en Enfermería es significativamente menor y en T.C.A.E. es superior. De los sujetos que participaron el $63.64 \%$ fueron Grado de Enfermería y el $36.36 \%$ T.C.A.E. El $76.22 \%$ estudió en La Provincia, mientras que el $23.78 \%$ lo hizo en otra distinta, Con respecto a la comunidad autónoma de nacimiento el $86.01 \%$ nació en Canarias y el $13.99 \%$ en otra diferente.

Teniendo en cuenta la distancia del hogar al puesto laboral el $29.37 \%$ es menor de 5 $\mathrm{Km}, 26.57 \%$ es entre 6 y $15 \mathrm{Km}$, el $23.08 \%$ es entre 15 y $25 \mathrm{Km}$, y el $20.98 \%$ es superior a $25 \mathrm{Km}$. En el desempeño de las labores del hogar el 55.24\% las comparte con otra o más personas y el $44.76 \%$ las realiza personalmente. Con respecto a otro puesto laboral el $95.1 \%$ no realiza otro trabajo y el $4.9 \%$ sí. En cuanto al grado de participación, el hospital que participó más fue el H.U.G.C. Dr. Negrín con un $63.64 \%$, mientras que en el C.H.U. Insular-Materno Infantil de Canarias fue un $36.6 \%$. En referencia a las unidades, la que más participación presentó fue quirófano con un $71.33 \%$, seguido R.E.A. con un $16.08 \%$, U.R.P.A. con $8.39 \%$ y C.M.A. $4.2 \%$. 


\section{DISCUSIÓN}

El porcentaje de respuesta es del $31.29 \%$ dato similar al $45.4 \%$ en un estudio ${ }^{(10)}$, y un $47.8 \%$ en otro estudio (11). Aunque hay estudios similares, hay otros donde este porcentaje es mucho mayor, pero hay que tener en cuenta que la población a estudio por la limitación geográfica sólo asciende a 457 sujetos.

En el último ítem de cuestionario sobre factores sociolaborales y demográficos, observamos que el $13.99 \%$ ha estado de baja laboral en los últimos 6 meses, frente al $86,01 \%$.

En cuanto a los factores sociodemográficos, podemos afirmar que, como se expuso anteriormente, se muestra el interés de los factores sociodemográficos del trabajador, ya que estos en diferente medida afecta al desempeño de las labores de Enfermería y T.C.A.E.

En el presente estudio se obtuvieron los siguientes datos, con respecto al grupo de edad predominantes es el situado entre 29 y 38 años con un $34.27 \%$, el $81.82 \%$ son mujeres, frente al $18.18 \%$ de hombres, este dato se extrapola a todas las áreas dentro de las profesiones de Grado de Enfermería y T.C.A.E., siendo profesiones con claro predomino del género femenino ${ }^{(12)}$, se comprobó que los efectos sobre la salud, en cuanto a la asociación entre variables "Conflicto-trabajo familiar", es un factor diferenciador entre ambos géneros, siendo para la muestra de mujeres los predictores más importantes, llegando a la conclusión de que el sector sanitario no se encuentra adaptado a las características emocionales de las mujeres debido sobre todo a las técnicas de liderazgo llevadas a cabo.

Referente a los años de experiencia la media es de 17.8 años y el tiempo de antigüedad 10.04 años. Este resultado es similar a un estudio ${ }^{(13)}$, donde se afirma que la antigüedad en el puesto de trabajo es debida al que el personal tiene que estar bien capacitado y requieren años de preparación para ese puesto de trabajo. Haciendo mención a los Servicios, el $71.33 \%$ de los participantes trabajan en Quirófano el $18.08 \%$ en R.E.A., el $8.39 \%$ en U.R.P.A. y el $4.2 \%$ en C.M.A. Cabe mencionar un estudio de Satisfacción Laboral y Estrategias de Cambio de las enfermeras en los Hospitales Públicos de Badajoz y Cáceres ${ }^{(14)}$, que expone como resultado que en las Unidades Quirúrgicas de los hospitales con mayor número de camas y enfermeras, presentan un mayor estrés.

Según el tipo de contrato, el $34.97 \%$ es fijo, 32.87 eventual, el $24.48 \%$ interino y el $7.69 \%$ sustituto. Comparando con otro estudio ${ }^{(15)}$, muestra que el $43.7 \%$ no tiene un puesto estable y en este estudio es más elevado, en un $65.03 \%$ y no dispone un contrato laboral fijo, dato muy relevante, ya que el tipo de contrato origina una estabilidad laboral y personal de gran envergadura en los trabajadores. Este porcentaje es tan elevado debido a que las oposiciones que se han realizado en la Comunidad, han estado muy distanciadas en el tiempo siendo la última convocatoria en el 2007 y resuelta en el 2020. Por lo que genera inestabilidad laboral además a este punto se suma la crisis económica que se generó en el país de 2008-2014 y que afecto a todos los sectores laborales. 
Señalando la rotación de puesto, el $55.24 \%$ trabaja en el mismo puesto, $37.06 \%$ rota dentro de la unidad, $7.69 \%$ rotan por otras unidades. El personal que rota es elevado $44.76 \%$, y no hemos encontrado estudios que relacione la rotación del puesto del trabajo con el estrés, por ello se aportan estos datos que podrían ser comparados con futuros estudios.

Teniendo en cuenta los factores sociodemográficos y laborales existe un estudio que analiza la importancia del contexto del trabajo ${ }^{(16)}$, donde sus resultados demuestran que los factores sociodemográficos y laborales influyen en la calidad laboral.

Existe un estudio sobre el Síndrome de Burnout en Profesionales de Enfermería de Cuidados Paliativos del País Vasco ${ }^{(17)}$, donde se realiza una comparación entre las enfermeras de Cuidados Paliativos y un grupo control de enfermeras de los mismos hospitales, pero en otras unidades de hospitalización y, como conclusión, aportó que los niveles "síndrome de estar quemado" de las enfermeras de los dos grupos eran similares. En nuestro estudio no se llegó a comparar con otras unidades, en contraposición un estudio indica que el personal de Enfermería tiene mayor estrés en este tipo de unidades específicas ${ }^{(18)}$. Para minimizar el estrés un estudio, refiere que el entrenamiento de estrategias de afrontamiento de estrés facilitaría a los profesionales enfermeros amortiguar y minimizar las consecuencias negativas de estrés laboral, siendo lo más perjudicial para los mismos la aparición del síndrome de burnout ${ }^{(19)}$.

En referencia a la diferenciación según sea el ámbito laboral cabe mencionar el estudio "Validation of moral distress instrument in nurse of primary health care" (20), dónde los resultados muestran que el instrumento es capaz de identificar los factores que desencadenan la angustia moral en las enfermeras de atención primaria, cuya limitación era el hecho de ser el primero en aplicarse y junto a otros sirve de precedente para la especificación según el ámbito laboral.

Hay un estudio actual "Factores laborales estresantes en el profesional de Enfermería y su influencia en la percepción de calidad de la atención a los pacientes del servicio de hospitalización clínica de Hospital General IESS Quito Sur, Quito" (21), en el que, aunque utilizaron otras herramientas para medir, las adaptaron y los resultados fueron específicos y hablan de la impotencia ante el caso de un paciente que no presenta mejoría, escasez de personal y dificultades al trabajar con un enfermero/a o enfermeros/as en particular, de su propia unidad.

Otro estudio que relaciona los estresores laborales y la percepción de la autoeficacia del cuidado humanizado, evidenció que en su muestra la variable de percepción de autoeficacia del cuidado humanizado era considerada como media, y en ningún miembro de la población llegó a alcanzarse una percepción alta ${ }^{(22)}$. Este resultado nos deriva a que el estudio del estrés laboral tiene como importancia su medición, ya que dependerá de la generalidad que queramos medir o el nivel de especificidad (23).

Por todo esto, el objetivo de este estudio se centraba en conocer el nivel de estrés del personal de Grado de Enfermería y T.C.A.E. e identificar si el estrés está asociado a las variables sociolaborales y demográficas de la muestra. 


\section{CONCLUSIONES}

En referencia al Estrés debemos señalar que, aunque a nivel general no presenta un nivel muy alto, hay varias cuestiones que debemos meditar y destacar, así en la tercera pregunta, en el último mes, "¿con qué frecuencia se ha sentido nervioso o estresado?", el $42.66 \%$ respondió "de vez en cuando", el $24.48 \%$ respondió "a menudo" y el $15.38 \%$ "muy a menudo". Estos niveles son elevados y denotan que es necesaria una intervención, que el personal esté nervioso o estresado, no es el entorno propio para dar una asistencia de calidad digna del cliente/paciente. Con respecto a las cargas de trabajo las siguientes preguntas nos sirven de referencia. La octava pregunta, en el último mes, "¿con qué frecuencia ha sentido que no podía afrontar todas las cosas que tenía que hacer?", el $38.46 \%$ respondió "de vez en cuando" y el $14.69 \%$ respondió "a menudo", son datos a tener en cuenta, aunque el $28.67 \%$ contestó "casi nunca". Y la decimosegunda pregunta, en el último mes, "¿con qué frecuencia ha pensado sobre las cosas que le quedan por hacer?", el $31.47 \%$ respondió "de vez en cuando", el $32.47 \%$ "a menudo" y el $25.87 \%$ "muy a menudo", niveles muy elevados que, aunque enfermería sea una profesión de continuidad de cuidados, denotan su complejidad, requieren de menor carga de trabajo y menores niveles de estrés.

Respecto a la asociación de la variable dependiente con las independientes, o sociolaborales y demográficas, existe una clara relación, las características individuales de cada trabajador como el género, experiencia laboral, edad, afectan significativamente a la variable dependiente de Estrés percibido.

Los resultados de nuestro estudio presentan un nivel de estrés con una mediana de $22 \%$. Con respecto a la edad de los encuestados oscila entre 29 y 64 años, siendo la media 43,57 años.

En cuanto a la localización de los encuestados, o lugar de su puesto de trabajo, el 63.64\% fue personal del H.U. Dr. Negrín, mientras que en el C.H.U. Insular-Materno Infantil de Canarias fue un $36.6 \%$. La mayoría de los participantes trabajaba en la unidad de Quirófano $71.33 \%$, y en la unidad de menor participación fue C.M.A. con un $4.2 \%$, siendo R.E.A. y U.R.P.A. donde se encuentran los valores intermedios $16.08 \%$ y $8.39 \%$ respectivamente. No hay evidencias para afirmar que las medianas en hombres y mujeres son distintas, ni entre los dos Hospitales Públicos, ni entre las dos titulaciones estudiadas, no hay una tendencia que a mayor o menor edad hay más estrés.

Como conclusión, es importante aportar los resultados a los Hospitales y analizar cómo se podría dar mejora a las dimensiones estudiadas, desarrollando estrategias que las disminuyan. En nuestra opinión se debería realizar controles periódicos de estos factores en las reuniones de las unidades. La finalidad es mejorar el entorno del profesional, que a su vez de traduce en una mejor calidad asistencial

\section{REFERENCIAS}

1. Organización Mundial de Salud, 1984.

2. Lazarus, R. y Folkman, S. Stress, appraisal and coping. New York: Springer. Publishing Company, 1984. 
3. Holmes, $T$ and Rahe, R. The social readjustment rating scale. J. Psychosom. Res, 1967; 1 1, 213-218.

4. Chorot, P. y Sandín, B. Life event and stress reactivity as predictors of cancer, coronary heart disease and anxiety disorders. International Journal of Psychosomatics, 1994; 41:34-40.

5. Pelechano, V., Matud, M.P. y de Miguel, A. Habilidades de afrontamiento en enfermos físicos crónicos, Análisis y Modificación de Conducta, 1993; 19, pp.91-149.

6. Anietei, M., Stoica, I. \& Samsonescu, M. Particularities of personality traits and perceived stress at workplace. For the young workers in Romania, 2013; 84, 10101014.

7. Cervantes, V.H. Interpretaciones del coeficiente alpha de Cronbach. Avances en Medición, 2005; 3, 9-28.

8. Cohen, S., Kamarck, T., \& Mermelstein, R. A global measure of perceived stress. Journal of Health and Social Behavior, 1983; 24, 385-396.

9. Benavides, F.G., Merino-Salazar, P., Cornelio, C., Vices, A. Cuestionario básico y criterios metodológicos para las Encuestas sobre Condiciones de Trabajo, Empleo y Salud en América Latina y el Caribe. Cad. Saúde Pública, Rio de Janeiro, 2016; vol. $32 \mathrm{~N}^{\circ}$ 9. En:

http://www.scielo.br/scielo.php?script=sci arttext\&pid=S0102311X2016000904004

10. Martín MC, Santiago I, Rodríguez F, Moreno MT, Andrés P, Casadevall A, et al. Estrés relacionado con el trabajo y exposición laboral en enfermeras de la Comunidad de Castilla y León. Enferm Clin., 2006; 16:119-26.

11. Grau A, Suñer R, García MM. Desgaste profesional en el personal sanitario y su relación con los factores personales y ambientales. Gac Sanit., 2005; 19:463-70.

12. Llorca Rubio, J.L. Tesis doctoral: Evaluación del Estrés Laboral, y sus Consecuencias en Trabajadores del sector sanitario, una perspectiva de género. Universidad de Valencia, 2017.

13. Cabana Salazar, J.A., García Ceballos, E., García Garcia, G.,Dávila Ramírez, R. El síndrome de Burnout en el personal de una unidad quirúrgica. Revista Médica Electrónica, 2009; V.31, N.3.

En: http://scielo.sld.cu/scielo.php?script=sci arttext\&pid=S168418242009000300004

14. Pablos González, M. del M. Tesis doctoral: Estudio de satisfacción laboral y Estrategias de Cambio de las Enfermeras en los Hospitales Públicos de Badajoz y Cáceres. Universidad de Extremadura, 2016.

15. Díaz Oviedo, Aracely. Adaptación y estrés laboral en el persona de las unidades médico-quirúrgicas. Revista iberoamericana de las Ciencias Sociales y Humanísticas. 2013; Issn-e 2395-7972, vol.2, n³. Pág 30-43. En: https://dialnet.unirioja.es/servlet/articulo?codigo $=5056006$

16. Lee JSY, Akhtar S. Effects of the workplace social context and job content nurse burnout. Human Resource Management, 2011; 50(2):227-245.

17. Martín García, M., Centeno Cortés, C., Sanz-Rubiales, A., Del Valle, M.L. Estudio sobre el Síndrome de Burnout en Profesionales de Enfermería de Cuidados Paliativos del País Vasco. Rev.Med. Universidad de Navarra, 2009; 53 (1):3-8.

18. Gómez Cantora. C. Tesis doctoral: Determinantes del Estrés Laboral Asistencial de Personal de Enfermería con enfermos Gerontológicos Terminales. Universidad de Santiago de Compostela, 2013.

19. Arrogante, O. Estrategias de afrontamiento al estrés laboral en Enfermería. Metas de Enfermería, 2016; $n^{\circ} 10$ volumen 19. En: https://www.enfermeria21.com/revistas/metas/articulo/81011/

20. Barth, P., Souza, F.R., Devos, E.L., de Lima, G., Ghizoni, D. Validation of a moral distress instrument in nurses of primary health care. Rev. Latino-A., 
Enfermagem, 2008; vol. $26 . \quad$ En: http://www.scielo.br/scielo.php?script=sci arttext\&pid=S010411692018000100320\&la ng=es

21. Irazabal, E.M. y Leano, C.E. Factores laborales estresantes en el profesional de Enfermería y su influencia en la percepción de calidad de atención a los pacientes del servicio de hospitalización General IESS Quito Sur, Quito. Facultad de Ciencias Médicas, 2009. En: http://www.dspace.uce.edu.ec/bitstream/25000/18518/1/T-UCE0014-ME072.pdf

22. Cabañas, I. y Delgado, J. Tesis: Estresores laborales y percepción de autoeficacia del cuidado humanizado en enfermeras(os), servicios de emergencia, Hospital III Yanahuara ESSALUD, 2017; En: http://repositorio.unsa.edu.pe/bitstream/handle/UNSA/4376/ENScaesid.pdf?sequence $=1$ \&isAllowed $=\mathrm{y}$

23. López Montesinos, M.J. Tesis doctoral: Consecuencias Psicosociales del trabajo en personal de Enfermería como indicadores de los Recursos Humanos. Universidad de Murcia, 2009. 